\title{
Atomic data for interpretation of HgMn spectra: Transition probabilities of $\mathrm{Mn}$ III $^{\star}$
}

\author{
P.H.M. Uylings and A.J.J. Raassen
}

Van der Waals-Zeeman Instituut, Universiteit van Amsterdam, Valckenierstraat 65-67, 1018 XE Amsterdam, The Netherlands

Received February 6; accepted March 18, 1997

\begin{abstract}
Transition probabilities of the spectrum of Mn III are calculated using the orthogonal operator description for both the odd and the even energy levels.
\end{abstract}

Key words: atomic data — star: chemically peculiar

\section{Introduction}

Chemically peculiar stars are known to display a high Mn abundance: manganese lines are strong in Bp stars, especially in the HgMn subgroup (Jaschek \& Jaschek 1995). Examples are $\kappa$ Cancri, HR 7245 and $\chi$ Lupi. Higher ionization will be found in Ap stars. While the $4 \mathrm{~s}-4 \mathrm{p}$ transitions of Mn III are certainly expected to appear e.g. in stellar Hubble (HST) spectra, most identification work up to now is restricted to Mn I and Mn II (Adelman et al. 1993; Wahlgren et al. 1994; Lopez-Garcia \& Adelman 1994; Wahlgren et al. 1995; Pintado \& Adelman 1996). It seems that a lack of knowledge of the Mn III spectrum frustrates the identification possibilities at the moment and therefore, the calculation of Mn III intensities appears a logical project to undertake. The $3 \mathrm{~d}-4 \mathrm{p}$ transitions of $\mathrm{Mn}$ III, however, fall below the HST $1200 \AA$ limit, but may in the near future be observed at high resolution by FUSELYMAN down to the onset of the Lyman continuum at $912 \AA$.

The present work is the second to calculate transition probabilities in the framework of orthogonal operators. It has been shown (Raassen \& Uylings 1996) to give reliable results for complex atoms, i.e. atoms with $Z>20$ and more than one electron outside closed shells. Characteristics of the method can be found in our recent

Send offprint requests to: P.H.M. Uylings

* The full Tables 2 and 4 are only available in electronic form at the CDS via anonymous ftp to cdsarc.u-strasbg.fr (130.79.128.5) or via http://cdsweb.u-strasbg.fr/Abstract. html publication on Ti III and V IV (Raassen \& Uylings 1997). In Sect. 2, a short introduction of the method is given. In Sect. 3, details of the calculation as well as the numerical results are presented.

\subsection{Orthogonal operators}

Theoretical transition probabilities can be obtained either from pure ab initio calculations or from an admixture of first principles theory and input of experimental energies. Examples of the first method are Multi-Configuration Hartree-Fock (MCHF, Froese Fischer 1978) or DiracFock (MCDF, Parpia et al. 1996), and the Configuration Interaction Version 3 code (CIV3, Hibbert 1975). The second, semi-empirical approach is more apt for the study of complex atoms, in view of the required eigenvector accuracy. The semi-empirical method consists of the following steps:

- Fitting eigenvalues of a model Hamiltonian containing adjustable parameters to the experimental energies.

- Obtaining eigenvectors from a diagonalization of this Hamiltonian at the end of the fitting procedure.

- Using theoretical (Hartree-Fock) values for the radial transition integrals to obtain line strengths in pure LS coupling.

- Transforming these LS values by means of the semiempirical eigenvectors to the final $\log (g f)$ or $A$-values.

In its original form this approach already has considerable success (Cowan 1981; Kurucz 1993), especially in the field of the heavier and more complex atoms where it is particularly powerful compared to other methods.

Subsequently, the introduction of orthogonal operators in the model Hamiltonian (Hansen et al. 1988), as a consequence of which the parameters become more stable and relatively independent of one another, turns out to raise the accuracy of the approach by up to an order of magnitude. The parameter independency offers the possibility to include additional operators that account for smaller effects like higher order or pure relativistic interactions.

While the method is semi-empirical, ab initio calculations are important in the procedure, both in obtaining 
Table 1. Values for the electric dipole transition integrals in Mn III calculated by means of MCDF including core polarization

\begin{tabular}{c|rccrc} 
& $3 \mathrm{~d}^{4} 4 \mathrm{p}$ & $3 \mathrm{~d}^{3} 4 \mathrm{~s} 4 \mathrm{p}$ & $3 \mathrm{~d}^{2} 4 \mathrm{~s}^{2} 4 \mathrm{p}$ & $3 \mathrm{~d}^{4} 5 \mathrm{p}$ & $3 \mathrm{~d}^{4} 4 \mathrm{f}$ \\
\hline $3 \mathrm{~d}^{5}$ & .729 & - & - & .192 & .349 \\
$3 \mathrm{~d}^{4} 4 \mathrm{~s}$ & -2.480 & .607 & - & .143 & - \\
$3 \mathrm{~d}^{3} 4 \mathrm{~s}^{2}$ & - & -2.280 & .515 & - & - \\
$3 \mathrm{~d}^{4} 4 \mathrm{~d}$ & -2.910 & - & - & 3.220 & -4.300 \\
$3 \mathrm{~d}^{4} 5 \mathrm{~s}$ & 1.570 & - & - & -5.360 & -
\end{tabular}

Table 2. Calculated $\log (g f)$ values for the $\left(3 \mathrm{~d}^{5}+3 \mathrm{~d}^{4} 4 \mathrm{~s}\right)-3 \mathrm{~d}^{4} 4 \mathrm{p}$ transition array of Mn III

\begin{tabular}{|c|c|c|c|c|c|c|c|}
\hline$\lambda(\AA)$ & $\log (g f)$ & $J_{\mathrm{f}}$ & $E_{\mathrm{f}}\left(\mathrm{cm}^{-1}\right)$ & even & $J_{\mathrm{i}}$ & $E_{\mathrm{i}}\left(\mathrm{cm}^{-1}\right)$ & odd \\
\hline 2433.471 & -.741 & 1.5 & $71564.21^{*}$ & $\left.\left.2\right|_{4} ^{5} \mathrm{D}\right)^{4} \mathrm{D}$ & .5 & 112645.31* & $\left.\left.1\right|_{4} ^{5} \mathrm{D}\right)^{4} \mathrm{P}$ \\
\hline 2423.720 & -.390 & 2.5 & $71831.98 *$ & $\left.2 \mid{ }_{4}^{5} \mathrm{D}\right)^{4} \mathrm{D}$ & 1.5 & $113078.34^{*}$ & $\left.\left.1\right|_{4} ^{5} \mathrm{D}\right){ }^{4} \mathrm{P}$ \\
\hline 2423.504 & -.705 & .5 & $71395.27^{*}$ & $\left.\left.2\right|_{4} ^{5} \mathrm{D}\right)^{4} \mathrm{D}$ & .5 & 112645.31* & $\left.\left.1\right|_{4} ^{5} \mathrm{D}\right){ }^{4} \mathrm{P}$ \\
\hline 2409.301 & -.241 & 3.5 & $72183.33^{*}$ & $\left.\left.2\right|_{4} ^{5} \mathrm{D}\right){ }^{4} \mathrm{D}$ & 2.5 & $113676.53^{*}$ & $\left.\left.1\right|_{4} ^{5} \mathrm{D}\right)^{6} \mathrm{D}$ \\
\hline 2408.086 & -.616 & 1.5 & $71564.21^{*}$ & $\left.2 \mid{ }_{4}^{5} \mathrm{D}\right)^{4} \mathrm{D}$ & 1.5 & $113078.34^{*}$ & $\left.\left.1\right|_{4} ^{5} \mathrm{D}\right)^{4} \mathrm{P}$ \\
\hline 2389.069 & -.757 & 2.5 & $71831.98 *$ & $\left.\left.2\right|_{4} ^{5} \mathrm{D}\right)^{4} \mathrm{D}$ & 2.5 & $113676.53^{*}$ & $\left.\left.1\right|_{4} ^{5} \mathrm{D}\right)^{6} \mathrm{D}$ \\
\hline 2374.314 & -.205 & 3.5 & $72183.33^{*}$ & $\left.\left.2\right|_{4} ^{5} \mathrm{D}\right)^{4} \mathrm{D}$ & 2.5 & 114287.91* & $\left.\left.1\right|_{4} ^{5} \mathrm{D}\right){ }^{6} \mathrm{D}$ \\
\hline 2365.414 & -.679 & 2.5 & $71831.98^{*}$ & $\left.2 \mid{ }_{4}^{5} \mathrm{D}\right){ }^{4} \mathrm{D}$ & 1.5 & $114094.97^{*}$ & $\left.\left.1\right|_{4} ^{5} \mathrm{D}\right)^{6} \mathrm{D}$ \\
\hline 2354.663 & -.865 & 2.5 & $71831.98^{*}$ & $\left.\left.2\right|_{4} ^{5} \mathrm{D}\right){ }^{4} \mathrm{D}$ & 2.5 & 114287.91* & $\left.\left.1\right|_{4} ^{5} \mathrm{D}\right)^{6} \mathrm{D}$ \\
\hline 2350.520 & -.972 & 1.5 & $71564.21^{*}$ & $\left.\left.2\right|_{4} ^{5} \mathrm{D}\right)^{4} \mathrm{D}$ & 1.5 & $114094.97^{*}$ & $\left.\left.1\right|_{4} ^{5} \mathrm{D}\right)^{6} \mathrm{D}$ \\
\hline 2238.026 & -.604 & 3.5 & $72183.33^{*}$ & $\left.\left.2\right|_{4} ^{5} \mathrm{D}\right)^{4} \mathrm{D}$ & 3.5 & $116851.69 *$ & $\left.\left.1\right|_{4} ^{5} \mathrm{D}\right)^{4} \mathrm{~F}$ \\
\hline 2228.457 & -.448 & 2.5 & $71831.98 *$ & $\left.\left.2\right|_{4} ^{5} \mathrm{D}\right)^{4} \mathrm{D}$ & 2.5 & $116692.14^{*}$ & $\left.\left.1\right|_{4} ^{5} \mathrm{D}\right)^{4} \mathrm{~F}$ \\
\hline 2227.451 & .428 & 3.5 & $72183.33^{*}$ & $\left.\left.2\right|_{4} ^{5} \mathrm{D}\right)^{4} \mathrm{D}$ & 4.5 & $117063.74^{*}$ & $\left.\left.1\right|_{4} ^{5} \mathrm{D}\right)^{4} \mathrm{~F}$ \\
\hline 2220.743 & -.537 & 1.5 & $71564.21^{*}$ & $\left.2 \mid{ }_{4}^{5} \mathrm{D}\right)^{4} \mathrm{D}$ & 1.5 & $116580.17^{*}$ & $\left.\left.1\right|_{4} ^{5} \mathrm{D}\right)^{4} \mathrm{~F}$ \\
\hline
\end{tabular}

Table 3. Values for the electric quadrupole transition integrals in Mn III calculated by means of MCDF including core polarization

\begin{tabular}{c|ccccc} 
& $3 \mathrm{~d}^{5}$ & $3 \mathrm{~d}^{4} 4 \mathrm{~s}$ & $3 \mathrm{~d}^{3} 4 \mathrm{~s}^{2}$ & $3 \mathrm{~d}^{4} 4 \mathrm{~d}$ & $3 \mathrm{~d}^{4} 5 \mathrm{~s}$ \\
\hline $3 \mathrm{~d}^{5}$ & 1.58 & -2.25 & - & -1.49 & 0.062 \\
$3 \mathrm{~d}^{4} 4 \mathrm{~s}$ & -2.25 & 1.34 & 1.24 & 8.57 & - \\
$3 \mathrm{~d}^{3} 4 \mathrm{~s}^{2}$ & - & 1.24 & 1.64 & - & - \\
$3 \mathrm{~d}^{4} 4 \mathrm{~d}$ & -1.49 & 8.57 & - & 1.30 & -24.3 \\
& - & - & - & 22.3 & - \\
$3 \mathrm{~d}^{4} 5 \mathrm{~s}$ & 0.062 & - & - & -24.3 & 1.30
\end{tabular}

initial estimates of the parameters (especially the parameters for the relativistic effects are sometimes left fixed at their ab initio value) and in finding the required radial transition integrals.

For more details on the method and for information on the parameter values and their behaviour, we refer to our recently published overview article on $\mathrm{d}^{N-1} \mathrm{p}$ configurations (Uylings \& Raassen 1996). Everybody interested in orthogonal operators is invited to contact the authors or to visit our Internet address ftp://nucleus.phys.uva.nl in the directory pub/orth.

\section{Transition probabilities}

To calculate the $\left(3 \mathrm{~d}^{5}+3 \mathrm{~d}^{4} 4 \mathrm{~s}\right) \rightarrow 3 \mathrm{~d}^{4} 4 \mathrm{p}$ transitions in $\mathrm{Mn}$ III properly, it is necessary to take into account the interaction with neighbouring configurations. For that reason the even system was built from $\left(3 d^{5}+3 d^{4} 4 s+3 d^{3} 4 s^{2}+3 d^{4} 4 d+3 d^{4} 5 s\right)$ and the odd system from $\left(3 d^{4} 4 p+3 d^{3} 4 s 4 p+3 d^{2} 4 s^{2} 4 p+3 d^{4} 5 p+3 d^{4} 4 f\right)$. Interactions with other (far-lying) configurations are taken into account by means of so-called effective operators. As outlined in the above, the full transition matrix is calculated after completing the fitting procedure. The angular part of the pure LS transition matrix is found from straightforward Racah algebra, while the radial 
Table 4. Calculated $A$-values for the $\left(3 \mathrm{~d}^{5}+3 \mathrm{~d}^{4} 4 \mathrm{~s}\right)-\left(3 \mathrm{~d}^{5}+3 \mathrm{~d}^{4} 4 \mathrm{~s}\right)$ M1 and E2 transition arrays of Mn III; the notation $x(y)$ means $x \times 10^{y}$

\begin{tabular}{ccccccccc}
$\lambda(\AA)$ & $A_{\mathrm{M} 1}\left(\mathrm{~s}^{-1}\right)$ & $A_{\mathrm{E} 2}\left(\mathrm{~s}^{-1}\right)$ & $J_{\mathrm{f}}$ & $E_{\mathrm{f}}\left(\mathrm{cm}^{-1}\right)$ & name & $J_{\mathrm{i}}$ & $E_{\mathrm{i}}\left(\mathrm{cm}^{-1}\right)$ & name \\
\hline 5588.382 & $3.61(-2)$ & $4.24(-2)$ & 2.5 & $51002.70^{*}$ & $\left.\left.1\right|_{5} ^{2} \mathrm{~F}\right)$ & 3.5 & $68892.00^{*}$ & $\left.\left.1\right|_{3} ^{2} \mathrm{G}\right)$ \\
5586.134 & - & $2.85(-3)$ & 2.5 & $51002.70^{*}$ & $\left.\left.1\right|_{5} ^{2} \mathrm{~F}\right)$ & 4.5 & $68899.20^{*}$ & $\left.\left.1\right|_{3} ^{2} \mathrm{G}\right)$ \\
5583.326 & - & $2.24(-3)$ & 1.5 & $43674.70^{*}$ & $\left.\left.1\right|_{3} ^{4} \mathrm{~F}\right)$ & 1.5 & $61580.20^{*}$ & $\left.\left.1\right|_{3} ^{2} \mathrm{D}\right)$ \\
5581.487 & $1.34(-3)$ & $1.30(-3)$ & 1.5 & $61580.20^{*}$ & $\left.\left.1\right|_{3} ^{2} \mathrm{D}\right)$ & 2.5 & $43668.80^{*}$ & $\left.1{ }_{3}^{4} \mathrm{~F}\right)$ \\
5574.142 & - & $3.38(-3)$ & 2.5 & $43668.80^{*}$ & $\left.\left.1\right|_{3} ^{4} \mathrm{~F}\right)$ & 2.5 & $61603.80^{*}$ & $\left.\left.1\right|_{3} ^{2} \mathrm{D}\right)$ \\
5544.587 & - & $1.93(-3)$ & 2.5 & $61603.80^{*}$ & $\left.\left.1\right|_{3} ^{2} \mathrm{D}\right)$ & 4.5 & $43573.20^{*}$ & $\left.1{ }_{3}^{4} \mathrm{~F}\right)$ \\
5411.274 & $2.10(-3)$ & $1.95(-3)$ & 1.5 & $61580.20^{*}$ & $\left.\left.1\right|_{3} ^{2} \mathrm{D}\right)$ & 2.5 & $43105.40^{*}$ & $\left.\left.1\right|_{3} ^{2} \mathrm{~F}\right)$ \\
5404.370 & $1.58(-3)$ & $1.98(-2)$ & 2.5 & $43105.40^{*}$ & $\left.\left.1\right|_{3} ^{2} \mathrm{~F}\right)$ & 2.5 & $61603.80^{*}$ & $\left.\left.1\right|_{3} ^{2} \mathrm{D}\right)$ \\
5347.422 & $1.87(-3)$ & - & 2.5 & $51002.70^{*}$ & $\left.\left.1\right|_{5} ^{2} \mathrm{~F}\right)$ & 3.5 & $32307.30^{*}$ & $\left.\left.1\right|_{5} ^{4} \mathrm{D}\right)$ \\
5268.987 & - & $2.47(-3)$ & 1.5 & $61580.20^{*}$ & $\left.\left.1\right|_{3} ^{2} \mathrm{D}\right)$ & 3.5 & $42606.50^{*}$ & $\left.\left.1\right|_{3} ^{2} \mathrm{~F}\right)$ \\
5262.441 & $3.61(-3)$ & - & 2.5 & $61603.80^{*}$ & $\left.\left.1\right|_{3} ^{2} \mathrm{D}\right)$ & 3.5 & $42606.50^{*}$ & $\left.\left.1\right|_{3} ^{2} \mathrm{~F}\right)$ \\
5086.087 & $6.82(-2)$ & - & 3.5 & $26859.90^{*}$ & $\left.\left.1\right|_{5} ^{4} \mathrm{G}\right)$ & 4.5 & $46515.90^{*}$ & $\left.\left.1\right|_{3} ^{2} \mathrm{H}\right)$ \\
\hline
\end{tabular}

transition integrals are obtained from a relativistic Hartree-Fock program (MCDF from Parpia et al. 1996). The values of the transition integrals, corrected for core polarization, are given in Table 1 . Subsequently, the pure LS transition matrix is transformed by the eigenvectors of the bra (even) and the ket (odd for E1, even for M1 or E2) system to obtain the transition probabilities between the initial and final states that are in reality expansions of pure LSJ states.

\subsection{E1 results}

In Table 2 the $\log (g f)$ values for the $\left(3 \mathrm{~d}^{5}+3 \mathrm{~d}^{4} 4 \mathrm{~s}\right) \rightarrow 3 \mathrm{~d}^{4} 4 \mathrm{p}$ electric dipole (E1) transitions are given. This system is selected by cutting off the higher energy values of both the even and the odd system in the final printing procedure; only $\log (g f)$ values larger than -1 are included. In this paper, a sample of the 14 highest lines is given; as explained in a footnote to the abstract, the complete table can be obtained at CDS.

The first column of this table shows the wavelength obtained from the energy differences between the experimental level values. Wavelengths below $2000 \AA$ are given as vacuum wavelengths and above $2000 \AA$ as air wavelengths. The second column gives the $\log (g f)$ values followed by the $J$-value, energy value and the name of the lower (even) level. The first character of the level name designates the configuration number: for the even levels " 1 " refers to $3 \mathrm{~d}^{5}$ and " 2 " to $3 \mathrm{~d}^{4} 4 \mathrm{~s}$; for the odd levels " 1 " refers to $3 \mathrm{~d}^{4} 4 \mathrm{p}$. An "** after the energy value indicates that the level is known, in which case the experimental level value is given. When unknown, the calculated energy value is given and used to approximate the wavelength. Full results including weaker lines and lines involving higher lying levels can be found on Internet.

\subsection{Forbidden lines}

Transition probabilities of forbidden, i.e. non-E1, transitions are only given for the lower even configurations, in view of their astrophysical relevance.

The radial part of the $\mathrm{E} 2$ transitions is, just as in the case of the E1 transitions, calculated from MCDF wavefunctions. In Table 3 the radial integrals for the electric quadrupole transitions are given in the form of a symmetric matrix. For E2-transitions within the $3 \mathrm{~d}^{4} 4 \mathrm{~d}$ configuration, there are two non-zero contributions, one for the $3 \mathrm{~d}-3 \mathrm{~d}$ and one for the $4 \mathrm{~d}-4 \mathrm{~d}$ transition. For this case, there are two rows in the table, the upper for the $3 \mathrm{~d}-3 \mathrm{~d}$ integral and the lower for the $4 \mathrm{~d}-4 \mathrm{~d}$ transition integral.

The $A$-values for the forbidden lines given in Table 4 , are restricted to the magnetic dipole (M1) and electric quadrupole (E2) transitions within the $3 \mathrm{~d}^{5}+3 \mathrm{~d}^{4} 4 \mathrm{~s}$ configurations, from levels with an energy of less than $75000 \mathrm{~cm}^{-1}$ above the ground and with $A$-values larger than $10^{-3} \mathrm{~s}^{-1}$. The level with the lower $J$-value is given first in the designation of the transition. Again, a sample of the table available at CDS is included in the paper.

\section{Conclusion}

With this work, we hope to provide some of the missing data for the interpretation of HgMn spectra. The present absolute transition probabilities of Mn III will be of interest in the region roughly between $900 \AA$ and $2000 \AA$. The $g f$-values of the $4 \mathrm{~s}-4 \mathrm{p}$ and $3 \mathrm{~d}-4 \mathrm{p}$ transitions of Mn III being available, identification and abundance determination of $\mathrm{Mn}$ in a range of chemically peculiar stars should now be facilitated. To avoid typing errors, all tables containing transition probabilities are computer processed. Complete results of the fits of both the odd and even energy system, as well as the corresponding complete transition arrays (without lower limits to the $g f$ - and $A$-values or restriction of the configurations) can be found in our 
database (anonymous ftp) at $\mathrm{ftp}: / /$ nucleus.phys.uva.nl in the directory pub/orth/mn3.

Acknowledgements. It is a pleasure to thank Dr. S. Johansson for discussing the astrophysical relevance of this work.

\section{References}

Adelman S.J., Cowley C.R., Leckrone D.S., Roby S.W., Wahlgren G.M., 1993, ApJ 419, 276

Cowan R.D., 1981, The Theory of Atomic Structure and Spectra. Berkeley, CA: University of California Press

Froese Fischer C., 1978, The Hartree-Fock Method for Atoms. New York: Wiley

Hansen J.E., Uylings P.H.M., Raassen A.J.J., 1988, Phys. Scr. 37,664

Hibbert A., 1975, Comput. Phys. Commun. 9, 141
Jaschek C., Jaschek M., 1995, The behaviour of Chemical Elements in Stars. Cambridge: Univ. Press

Kurucz R.L., 1993, Phys. Scr. T47, 110, URL address: http://cfa-www.harvard.edu/amp/data/ stats/kurucz.html

Lopez-Garcia Z., Adelman S.J., 1994, A\&AS 107, 353

Parpia F.A., Froese Fischer C., Grant I.P., 1996, Comput. Phys. Commun. 94, 249

Pintado O.I., Adelman S.J., 1996, A\&AS 118, 283

Raassen A.J.J., Uylings P.H.M, 1996, Phys. Scr. T65, 84

Raassen A.J.J., Uylings P.H.M., 1997, A\&AS 123, 47

Uylings P.H.M., Raassen A.J.J., 1996, Phys. Scr. 54, 505

Wahlgren G.M., Adelman S.J., Robinson R.D., 1994, ApJ 434, 349

Wahlgren G.M., Leckrone D.S., Johansson S.G., Rosberg M., Brage T., 1995, ApJ 444, 438 